\title{
Small animal PET based on 16x16 TSV-MPPCs and monolithic crystals
}

\author{
Antonio Gonzalez ${ }^{1 *}$, Albert Talens Aguilar ${ }^{1}$, Pablo Conde ${ }^{1}$, Liczandro Hernadez Hernadez ${ }^{1}$, \\ Luis Fernando Vidal San Sebastian', Carlos Correcher Salbador², Cesar Molinos Solsona², Sven Junge ${ }^{3}$, \\ Konrad Lankes ${ }^{3}$, Jose Maria Benlloch ${ }^{1}$
}

From PSMR 2015: 4th Conference on PET/MR and SPECT/MR

La Biodola, Isola d'Elba, Italy. 17-21 May 2015

${ }^{1}$ Institute for Instrumentation in Molecular Imaging, i3M-CSIC, Valencia, Spain
In this work we present the design of a small animal PET based on 8 high-density arrays of MPPCs and monolithic scintillators. The MPPCs arrays are composed of 16x16 TSV-type $\left(3 \times 3 \mathrm{~mm}^{2}\right)$ elements covering a rough active area of $5 \times 5 \mathrm{~cm}^{2}$. A single LYSO block with a thickness of $10 \mathrm{~mm}$ has been mounted on each detector. Black paint has been applied to the entrance and lateral faces of the crystal to preserve the scintillation light distribution. The axial and transaxial FOVs of one ring are $48 \mathrm{~mm}$ and $80 \mathrm{~mm}$, respectively. Each MPPC array has been directly attached to a resistive readout circuit that provides outputs for each row and column of the array. These 32 signals are read with flexible boards $30 \mathrm{~cm}$ apart from the PET detector without any additional connectors in between. The PET-system is intended for in-line acquisition in front of MR scanners and as PET-insert inside the sensitive MRI volume. For this purpose, it is necessary to avoid magnetic sensible materials, such as nickel, and to prevent eddy currents in metallic structures induced by the MRI gradients. All detectors are air cooled and kept at temperatures of approximately $20^{\circ} \mathrm{C}$ with a variation below $0.05^{\circ} \mathrm{C}$. The intrinsic resolution is $2.2 \mathrm{~mm}$ at the crystal center (averaged over all $2.6 \mathrm{~mm}$ ) when Center of Gravity methods are used to resolve the impact position. This value is about a factor 1.5 better than results obtained with the H8500 PSPMT (64 PADs) and similar scintillators. With an improved collimator with holes with only $0.8 \mathrm{~mm}$ diameter and a length of $70 \mathrm{~mm}$, an intrinsic detector resolution of $1.1 \mathrm{~mm}$ was reached. The energy resolutions of ROIs of $1 \times 1 \mathrm{~cm}^{2}$ showed FWHM values in the range of $14 \%$.

Authors' details

${ }^{1}$ Institute for Instrumentation in Molecular Imaging, i3M-CSIC, Valencia, Spain. ${ }^{2}$ Oncovision, Valencia, Spain. ${ }^{3}$ Bruker BioSpin, Germany.

Published: 18 May 2015

doi:10.1186/2197-7364-2-S1-A16

Cite this article as: Gonzalez et al.: Small animal PET based on 16x16 TSV-MPPCS and monolithic crystals. EJNMMI Physics 2015 2(Suppl 1):A16.

\section{SpringerOpen ${ }^{\odot}$}

(C) 2015 Gonzalez et al; licensee Springer. This is an Open Access article distributed under the terms of the Creative Commons Attribution License (http://creativecommons.org/licenses/by/4.0), which permits unrestricted use, distribution, and reproduction in any medium, provided the original work is properly cited. 